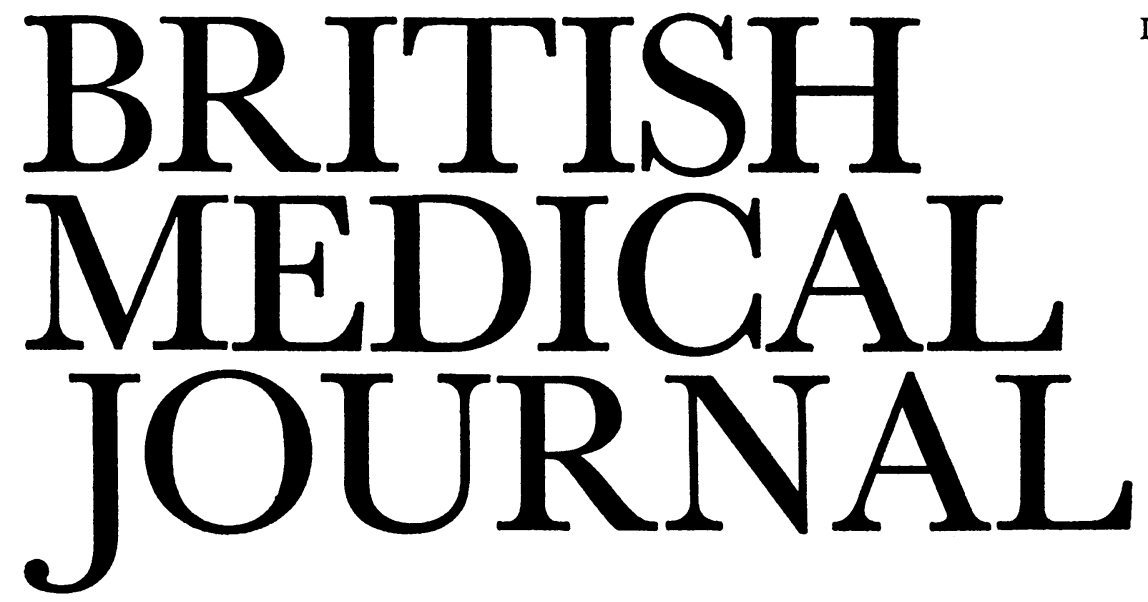

LONDON SATURDAY 23 DECEMBER 1972

\title{
The Present Influenza Outbreak
}

One of the principal factors to be considered in the development of a viral vaccine is its antigenic composition. Fortunately many of the common and severe virus diseases-for example, measles, smallpox, yellow fever, and pollomyelitis - are caused by viruses consisting of a single or closely related antigenic types. Furthermore, these viruses do not undergo antigenic variation. The same is true for rubella and mumps. As a result manufacturers and licensing authorities can say with reasonable assurance that the vaccines against them are likely to be as effective in the future as they are today. The reverse is the case with influenza, particularly the A strains, which are responsible for the most severe forms of the disease and the most extensive epidemics.

No other virus behaves quite like influenza in its ability to undergo periodic mutation. This fact first came to light in 1947, when it was found that vaccines prepared from influenza viruses prevalent during the 1940 s failed to protect against the new strain, termed A-prime, which emerged in 1946-7. A similar event occurred in 1957 when the Asian strain appeared on the scene. Much of our present knowledge of the behaviour of the influenza virus in terms of antigenic variation has come from the careful surveillance carried out by the World Health Organization and the World Influenza Centre.

The influenza A strains consist of three main subtypes: A0, prevalent from about 1933 to 1946 ; A1 from 1947 to 1957; and the A2 strain, from 1957 onwards. Influenza viruses contain three virus-specific antigens. The ribonucleoprotein is specific to the influenza viruses, while the two surface antigens, the haemagglutinin (HA) and the neuraminidase (NA), which are components of the viral envelope, are specific for the subtype or strain. In 1968 a new strain, A/Hong Kong/68, appeared and caused epidemics throughout the world until the winter of 1971-2. This strain contained an HA antigen distinct from that of the Asian strain, though its neuraminidase antigen was related to the Asian strain. In January this year one strain out of 752 examined in England showed altered antigenic characteristics from the A/Hong Kong/68 strain. Between May and September this new strain, designated A/England/42/72, was isolated from cases of epidemic influenza in the Far East, and it is the virus causing most if not all cases in Great Britain at present. ${ }^{1}$ The changes in antigenic composition of the new strain involved both the haemagglutinin and neuraminidase anti- gens, but both antigens belong to the same general subtype as the prototype strain A/Hong Kong/68. This antigenic change would, therefore, come into the category of antigenic "drift" rather than major "shift."

Antigenic variation, whether "drift" or "shift," raises practical problems in the production of vaccines. In 1957 vaccines containing the A-prime or A1 strains were considerably less effective in protecting against Asian influenza than those containing the A2 Asian strain. Past experience, therefore, has shown that unless the vaccine strain is closely related to the epidemic strain the degree of protection will be low. In practice this means that any new virus has to be isolated, identified, and then adapted to growth in fertile eggs for production of vaccine. Thus an influenza epidemic often appears before sufficient vaccine is available. The recent work of E. D. Kilbourne ${ }^{2}$ in the United States on the genetic recombination of influenza viruses has important practical implications. He has shown that it is possible in the laboratory to produce genetic combinations between different strains of influenza $A$ virus. If this technique is used with a new variant which grows poorly and a well-established strain which grows well, it is possible in a comparatively short time to produce large quantities of high-titre influenza virus fluid for vaccines. This in fact is no: being done with the A/England/42/72 strain, as reported in these columns a few weeks ago. ${ }^{3}$

Until the new vaccine is available in sufficient quantities, we have to consider the efficacy of those at present available containing the A/Hong Kong/68 strain. Marguerite Pereira, of the Central Public Health Laboratory, Colindale, London, and her colleagues at the World Influenza Centre and World Health Organization have recently studied the immunological response of volunteers given the current vaccine, and their report appears in the B.M.F. this week (page 701). Surprisingly, the response was much better than was expected in that $63 \%$ of the persons vaccinated developed antibody to the A/England/42/72 virus compared with $98 \%$ to the A/Hong Kong/68 strain. Such a response should confer some degree of protection, but nevertheless as soon as the new vaccine is available it should be used for people at special risk of serious complications of influenza. For them it should have a booster effect.

An important practical development in the influenza field is the production of purified vaccine by zonal centrifugation. 
This technique has led to a reduction in the toxicity which was such a prominent feature of earlier vaccines, certainly those used while Asian influenza was epidemic and thereafter.

In recent years the evidence has grown that changes in human influenza may originate in swine and other animals. Speculation has long centred on the close association in time between the great pandemic of influenza in 1918-19 and the epidemic of swine influenza which occurred in the middlewest of the United States. What is not clear is whether the swine developed influenza from man or the other way round. But worldwide studies have now shown that domestic fowls, ducks, and other birds and horses have influenza viruses of their own, some of which are related to those which infect man. For example, the A/Hong Kong/68 strain shares an antigen with the $\mathrm{A} 2$ equine virus, and there is also evidence of an antigenic relationship between duck influenza virus and human strains. Thus it is possible that genetic recombination may occur in nature as well as in the laboratory, and epidemics may be the result of genetic interaction between human and animal or avian influenza strains.

1 Public Health Laboratory Service, Communicable Disease Reports, 1972, No. 47.

${ }^{2}$ Kilbourne, E. D., Bulletin of the World Health Organization, 1969, 41, 643.

3 British Medical fournal, 1972, 4, 251.

\section{Diet and Congenital Defects}

Anencephalus and spina bifida share many epidemiological features and are together the commonest of the serious malformations in man. Consequently any theory about their aetiology which also suggests a method of prevention deserves careful consideration. Much publicity has been given to a hypothesis put forward by J. H. Renwick ${ }^{1}$ that these defects are caused by eating (or possibly peeling or cooking) blighted potatoes in early pregnancy. Specific but unidentified substances are invoked which may be present not only in potatoes affected by the blight fungus (Phytophthora infestans) but perhaps also in blight-resistant ${ }^{2}$ or even damaged $^{3}$ tubers, and particularly those that have been stored over winter. The evidence for the hypothesis demands careful appraisal.

There is no doubt that Ireland grows a lot of potatoes, has severe blight, and also the highest recorded incidence of anencephaly and spina bifida in the world. In addition the cultivation of potatoes, blight, and these malformations tend to become less frequent in an easterly direction across Europe or a westerly direction across the United States. Within Ireland, however, low anencephalic rates are recorded in the north-west and in parts of Ulster, ${ }^{4}$ where blight is severe. ${ }^{5}$ To explain this discrepancy Renwick suggested under-reporting from these areas.

In many other countries incidence rates of these defects are known only for hospital births, ${ }^{6}$ and the rates are liable to be inflated by the selective admission of mothers with a higher than average risk of having a baby with this type of deformity. Nevertheless, all available evidence suggests that France has a low incidence of these defects ${ }^{7-9}$ though potato consumption is high (464 lb (210 kg) per head in $1966 \mathrm{com}-$ pared to $266 \mathrm{lb}(120 \mathrm{~kg})$ in the U.K. $)^{10}$ and blight is severe in many areas. ${ }^{5} \mathrm{~A}$ similar contrast appears to exist in Sweden. ${ }^{510}$ On the other hand, potatoes form a small proportion of the diet in India and Nigeria, where there is an appreciable incidence of these defects among hospital births. ${ }^{611}$ Taiwan also has an appreciable incidence of neural-tube defects ( 1.42 per 1,000 total births, and higher in the poor) but very low potato consumption (about $1 \mathrm{lb}$ $(0.45 \mathrm{~kg})$ per head per year), and this is probably confined in the main to foreign residents and other well-off groups. ${ }^{10}$ Renwick has suggested that here these defects are caused by substances in rice produced in response to infection in the rice plant. ${ }^{12}$

A temporal correlation was claimed mainly because anencephalic stillbirth rates in Scotland as a whole ${ }^{13}$ correlated with blight data from the west of Scotland over the period 1946-55.5 Renwick explained the lack of correlation with blight in the east or north of Scotland on the grounds that "blight control is taken seriously" there. ${ }^{14}$ But in fact severe blight was recorded (on tubers and haulm) in more of these years than in the west, ${ }^{5}$ which also receives large quantities of potatoes from the east, the major (seed and non-seed) potato growing area in Scotland. ${ }^{15}$ Other data offered to support a secular link consisted in a small series of anencephalic births in Leicester ${ }^{16}$ which showed some correlation with blight in the Fens, which supply the Midlands with potatoes. The correlation of an even smaller series from Exeter with blight in the Fens had doubtful validity since many potatoes eaten there are grown locally. ${ }^{15}$ Other published series were not analysed because they related to the year of birth and not to the year of supposed initiation of the malformation, though a series of babies (stillbirths excluded) notified as born with spina bifida in west Scotland in 1952-6 $6^{18}$ was accepted and did correlate with blight there two years previously. 1219

Renwick explains the relationship between these neuraltube defects and low socio-economic group by reference to the greater consumption of potatoes by the less well-off, and the increased incidence among multiparous women by their exposure to the larger number of potatoes needed for a larger family. The high incidence reported in young primigravidae is explained by their greater likelihood to be living early in pregnancy in the parental home, where they will be exposed to more potatoes than if they were living independently. Similarly, the recent decline in frequency of these malformations is attributed to the success of cropspraying against blight, and the peak conception period in winter (in several series) to the consumption of imperfect potatoes harvested some months before.

The recent report of cranial anomalies in the fetuses of marmosets which had been fed on blighted potates ${ }^{20}$ is at first sight so arresting that it is tempting to overlook some of the inconsistencies in the potato blight hypothesis. Six conjugal pairs of marmosets were fed $8 \mathrm{~g}$ of freeze-dried "blighted" potato concentrate in addition to their normal diet and subjected to hysterotomy after an estimated 80-120 days of pregnancy. Cranial osseous defects were found in 4 of the 11 resulting fetuses. No such abnormalities were noted in the 11 young born to 6 control females which received a normal diet. The defects were most severe in those animals with the largest pre-conception exposure to the blighted potato concentrate (and these were also the youngest fetuses at hysterotomy). But it must be noted that these anomalies were neither anencephaly nor spina bifida. The marmoset is claimed to be a suitable model for the study of human teratogenesis on the grounds that it seems to respond to thalidomide like man, ${ }^{21}$ but experience with this animal in this field is still very limited.

The recent debate has directed attention to the subject of diet in general as a possible factor in the aetiology of these 